\title{
Unusual behaviour of the IGRF during the 1945-1955 period
}

\author{
Wen-yao Xu \\ Institute of Geophysics, Chinese Academy of Sciences, Beijing 100101, China \\ (Received February 4, 2000; Revised July 12, 2000; Accepted July 12, 2000)
}

\begin{abstract}
The International Geomagnetic Reference Field (IGRF) for 1900-2000 is tested in this paper. A peculiar behaviour is found in the time variations of higher-degree coefficients of the IGRF models. During the 1945-1955 period the spherical harmonic coefficients $g_{n}^{m}$ and $h_{n}^{m}$ with $n>7$ show unusual jumps, while their variations before and after this period are quite smooth. These irregular variations have little effect on the main features of the surface magnetic pattern. However, when we extrapolate the field pattern downward through the insulating mantle to the core-mantle boundary (CMB), the contributions of the higher-degree coefficients become more important and are likely to affect the shape of the geomagnetic energy spectrum, distribution of magnetic flux, and magnetic determination of the conducting core radius. It seems necessary to re-examine and revise these coefficients in the IGRF models.
\end{abstract}

\section{Introduction}

Since the adoption of the first International Geomagnetic Reference Field (IGRF) model, designated IGRF 1965, by IAGA in 1968 (IAGA Commission 2 Working Group 4, 1969; Zmuda, 1971), the IGRF has been revised seven times (IAGA Division 1 Study Group on Geomagnetic Reference Field, 1975; IAGA Division 1 Working Group 1, 1981, 1985, 1987; IAGA Division 5 Working Group 8, 1991, 1996, 2000). The historical development of the IGRF has been described in detail by Langel (1992) and Barton (1997). Charts and tables of gridded values of the IGRF were published as IAGA Bulletins before the seventh generation of IGRF (Baldwin and Langel, 1993), and from then on have been replaced by IGRF software for desktop computers (Macmillan and Barton, 1997).

The purpose of the IGRF models, as described by Zmuda (1971), is to form an agreed basis for main field calculation and to unify results in studies on, for example, removal of trend to yield surface anomalies, field residuals potentially applicable to the calculation of ionospheric and magnetospheric currents, the shape of a field line, locations of conjugate points, and field values used in the B-L space of trapped particles. Geomagnetic main field models are also an important database for studying secular variation of the main geomagnetic field and core dynamics (Bloxham and Gubbins, 1985; Bloxham and Jackson, 1991; Olson and Aurnou, 1999).

In this paper the IGRF for 1900-2000 is examined. The IGRF comprises discrete main-field models at 5-year intervals; those for 1945-1990 are definitive (DGRFs), and the rest are non-definitive (IGRFs). The time variations of Gauss coefficients during the 100-year period are shown in Section 2, the magnetic spectra at the earth's surface and core-mantle boundary are compared in Section 3, unsigned

Copy right (C) The Society of Geomagnetism and Earth, Planetary and Space Sciences (SGEPSS); The Seismological Society of Japan; The Volcanological Society of Japan; The Geodetic Society of Japan; The Japanese Society for Planetary Sciences. magnetic flux is calculated for different depths in Section 4, and finally, in Section 5 we test the influence of high-degree Gauss coefficients on the determination of the core radius by using magnetic-field models.

\section{Temporal Changes of IGRF Gauss Coefficients}

The IGRF is a series of spherical harmonic models of the Earth's main magnetic field and its secular variation (Chapman and Bartels, 1940; Langel, 1987). The eighth generation of IGRF comprises 21 main-field models at epochs 5 years apart from 1900 to 2000, and a secular-variation model for 2000-2005. Each main-field model has 120 spherical harmonic (or Gauss) coefficients, $g_{n}^{m}$ and $h_{n}^{m}$, and extends to degree and order 10. The secular-variation model has 80 coefficients and extends to degree and order 8 . The geomagnetic potential in the IGRF models is given by

$$
\begin{aligned}
V= & a \sum_{n=1}^{N} \sum_{m=0}^{n}\left(\frac{a}{r}\right)^{n+1} \\
& \cdot\left(g_{n}^{m} \cos m \varphi+h_{n}^{m} \sin m \varphi\right) P_{n}^{m}(\cos \theta)
\end{aligned}
$$

where $a$ is the mean radius of the earth, $r$ is radial distance from the earth's centre, $\varphi$ is longitude eastward from Greenwich, $\theta$ is geocentric colatitude, and $P_{n}^{m}(\cos \theta)$ is the associated Legendre function of degree $n$ and order $m$, normalised according to the convention of Schmidt. $N$ is the maximum spherical harmonic degree of the expansion, or truncation level.

Barton (1997) showed the time variations of the Gauss coefficients up to $n=m=8$. In order to check the rest of the coefficients, Fig. 1 shows the time variations of all 120 coefficients $g_{n}^{m}$ and $h_{n}^{m}$ in the IGRF models during the period 1900-2000.

As noted by Barton and seen in Fig. 1(a), the coefficients $g_{n}^{m}$ and $h_{n}^{m}$ change smoothly up to $n=5$. However, some steps between 1940 and 1950 are detectable in $g_{6}^{2}, g_{6}^{4}, h_{6}^{3}, h_{6}^{5}$, and $h_{6}^{6}$. 

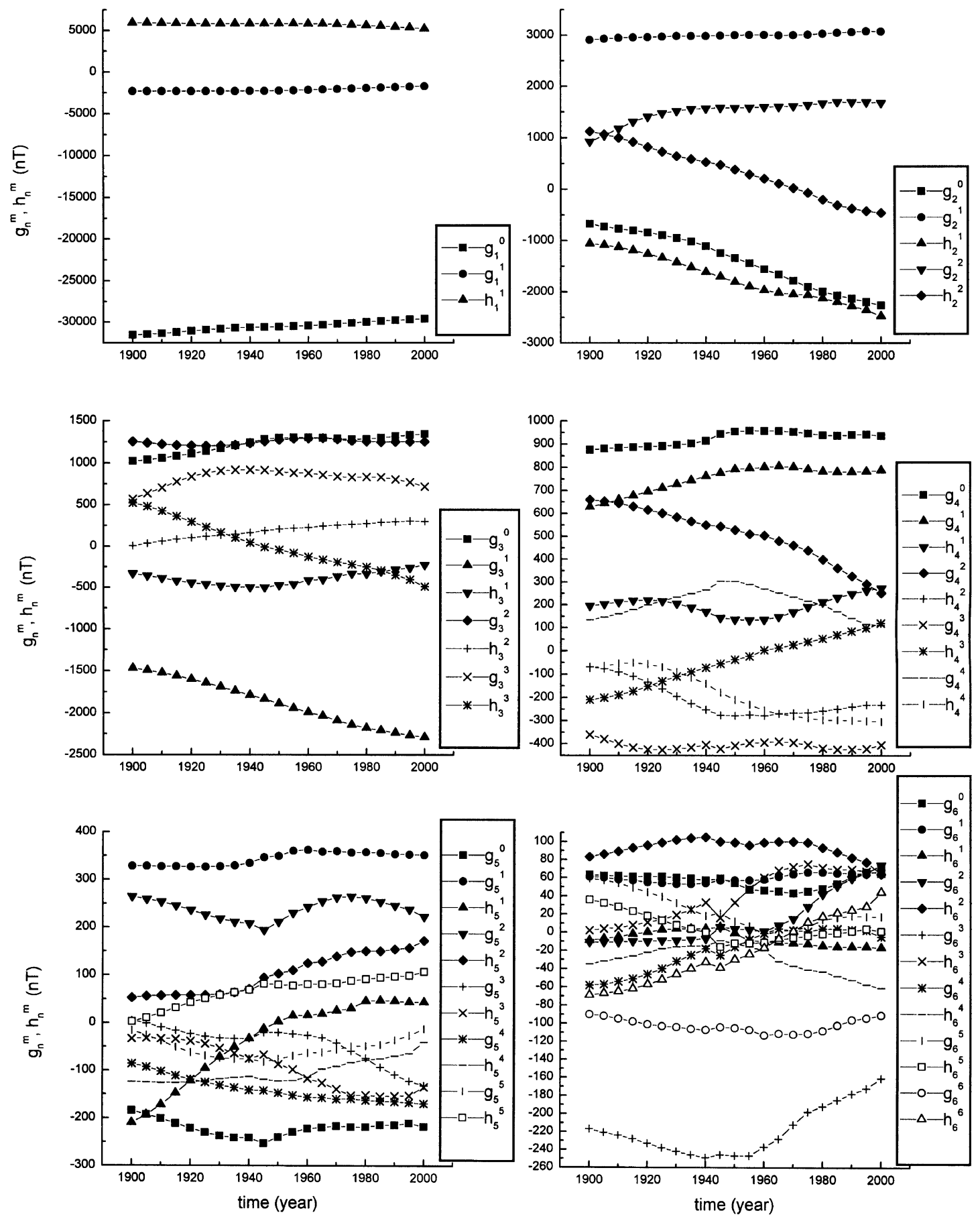

Fig. 1(a). Variations of Gauss coefficients $g_{n}^{m}$ and $h_{n}^{m}$ in the IGRF models for 1900-2000, $n=1-6$.

More prominent jumps can be seen in $g_{n}^{m}$ and $h_{n}^{m}$ with $n=$ 7 during the period 1945-1950 (Fig. 1(b)). As $n$ increases, jumps become dramatic, and the time span of jumps expands. For example, in Fig. 1(c) coefficient $g_{9}^{1}$ suddenly changes from $10 \mathrm{nT}$ for 1900-1940 to $-21 \mathrm{nT}$ for 1945, and then quickly recovers to $9 \mathrm{nT}$ in $1955 ; h_{9}^{3}$ increases from $5 \mathrm{nT}$ for 1900-1940 to $29 \mathrm{nT}$ for 1945 , and then decreases to $7 \mathrm{nT}$ in $1955 ; h_{10}^{3}$ changes from $2 \mathrm{nT}$ for $1900-1940$ to $-20 \mathrm{nT}$ for 1945 , and then gradually recovers to $0 \mathrm{nT}$ in 1960 . All of these step variations are limited to a relatively short time span, namely 1945-1955.
These irregular variations in higher-degree Gauss coefficients have little effect on the main features of the surface magnetic pattern (Barton, 1997). However, when we extrapolate the field pattern downward through the insulating mantle to the core-mantle boundary, the contributions of the higher-degree coefficients become more and more important, that they are likely to affect the shape of the geomagnetic energy spectrum, distribution of magnetic flux, magnetic determination of the conducting core radius, and the pattern of fluid flow on CMB. 

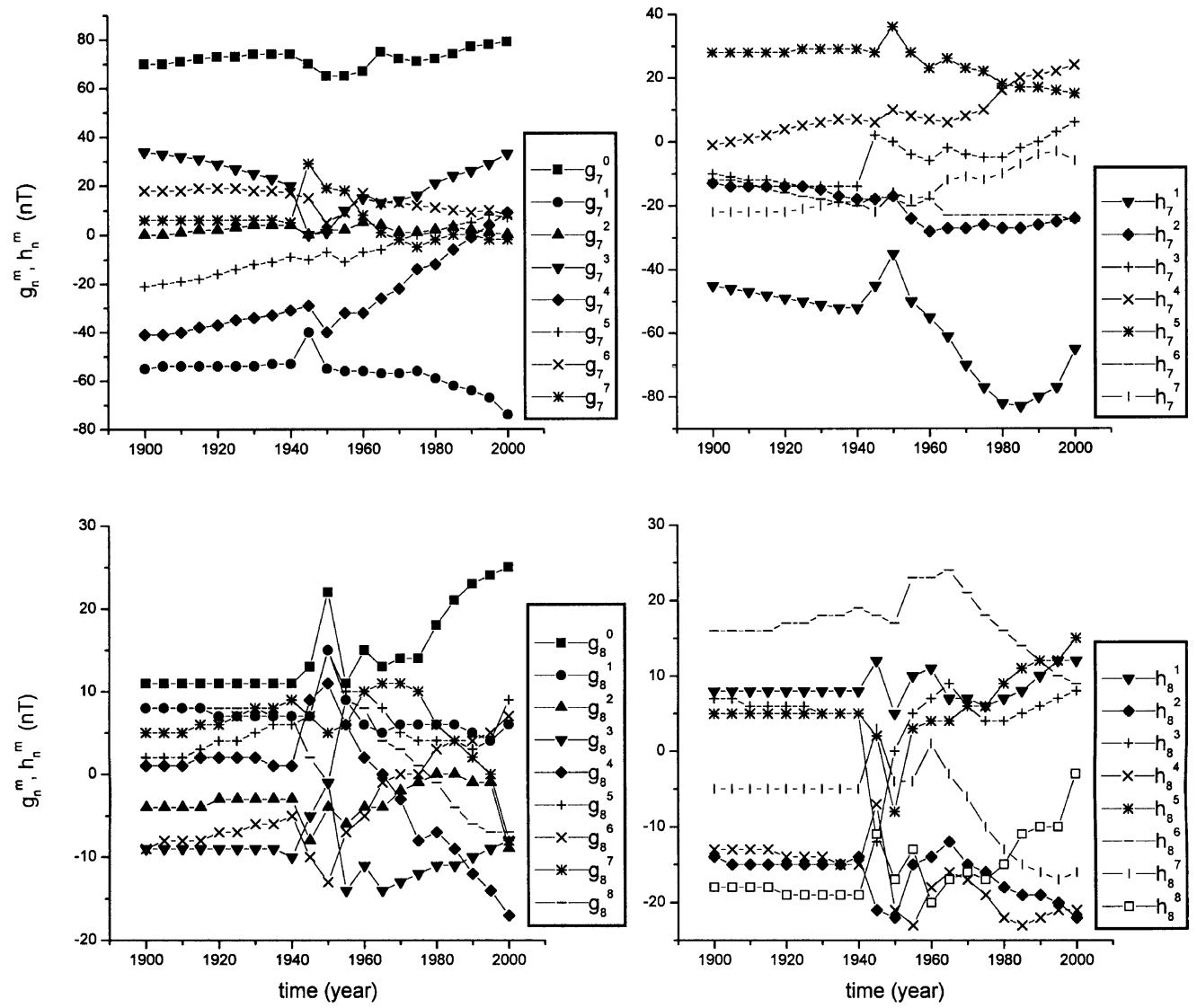

Fig. 1(b). Variations of Gauss coefficients $g_{n}^{m}$ and $h_{n}^{m}$ in the IGRF models for 1900-2000, $n=7$ and 8.
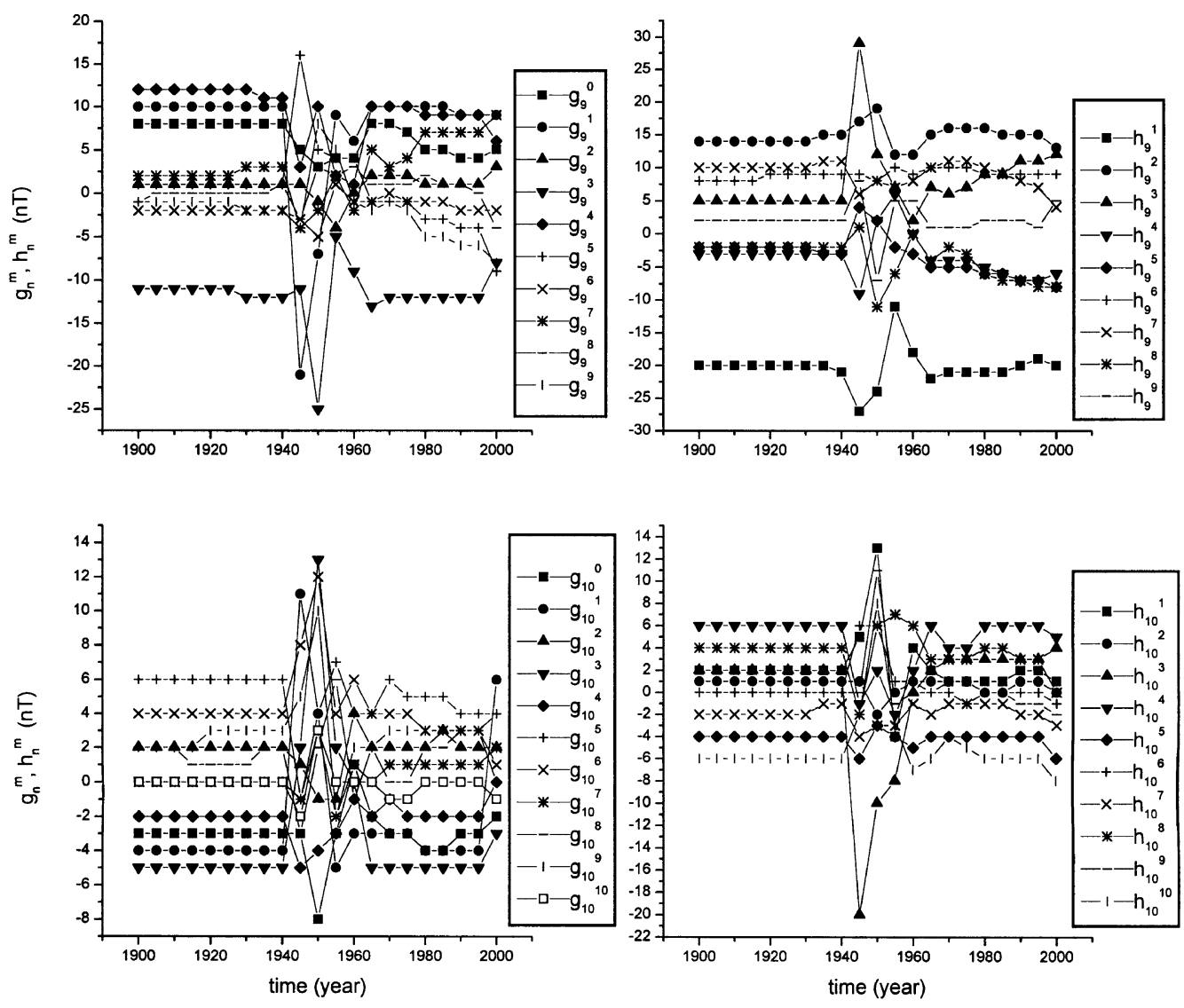

Fig. 1(c). Variations of Gauss coefficients $g_{n}^{m}$ and $h_{n}^{m}$ in the IGRF models for 1900-2000, $n=9$ and 10. 


\section{Anomalous Behaviour in the Geomagnetic En- ergy Spectrum}

The energy characteristics of the earth's main magnetic field is commonly described by the geomagnetic energy spectrum. The total magnetic energy external to the earth's core (radius $r=b$ ) can be expressed as follows (Verosub and Cox, 1971; Benton et al., 1982)

$$
M(b, t)=\frac{1}{2 \mu_{0}} \int_{b}^{\alpha} \int_{0}^{2 \pi} \int_{0}^{\pi} B^{2}(r, \theta, \varphi, t) r^{2} \sin \theta d \theta d \varphi d r .
$$

Using (1) and $B=-\nabla V$ gives

$$
\begin{aligned}
M(b, t)= & \sum_{n=1}^{N} M_{n}=\sum_{n=1}^{N} \frac{2 \pi a^{3}}{\mu_{0}} \frac{n+1}{2 n+1}\left(\frac{a}{b}\right)^{2 n+1} \\
& \cdot \sum_{m=0}^{n}\left[\left(g_{n}^{m}\right)^{2}+\left(h_{n}^{m}\right)^{2}\right]
\end{aligned}
$$

where $M_{n}$ is the energy related with the harmonics of degree $n$. A little different form is sometimes used for the 'spatial power spectrum' (Lowes, 1966, 1974; Langel and Estes, 1982)

$$
R_{n}=(n+1) \sum_{m=0}^{n}\left(\frac{a}{b}\right)^{2 n+4}\left[\left(g_{n}^{m}\right)^{2}+\left(h_{n}^{m}\right)^{2}\right]
$$

in which the lack of the factor $(2 n+1)^{-1}$, compared with $M_{n}$ in Eq. (3) makes it converge more slowly than does the series in (3). Besides, some other different factors proceeding the sum square of the spherical harmonic coefficients and different exponents of $(a / b)$ are used (Cain et al., 1989). Displays of $\log M_{n}$ (Joules) vs. degree $n$ at the ground surface $(r=a=6371 \mathrm{~km})$ and the CMB $(r=b=3480 \mathrm{~km})$ for different epochs of 1900-2000 are given in Fig. 2, where only the spectra for 1945 and 1950 are marked. The spectrum can be approximated by a straight line and this indicates the relative importance of the different harmonics in the total magnetic energy. The spectrum varies only slightly with time except $M_{9}$ and $M_{10}$ for 1945 and 1950, which show much larger strength compared with other epochs.

In order to show more clearly the unusual variation of $M_{9}$ and $M_{10}$, the ratios of $M_{n}(b, t) / M_{n}(b, 1900)$ are plotted

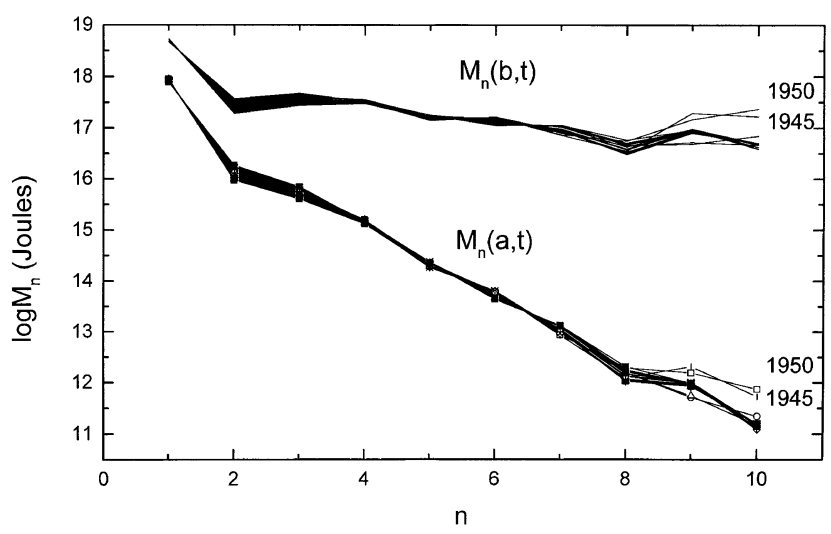

Fig. 2. Geomagnetic energy spectra at the ground surface $(r=a)$ and the CMB $(r=b)$ for different epochs of 1900-2000.

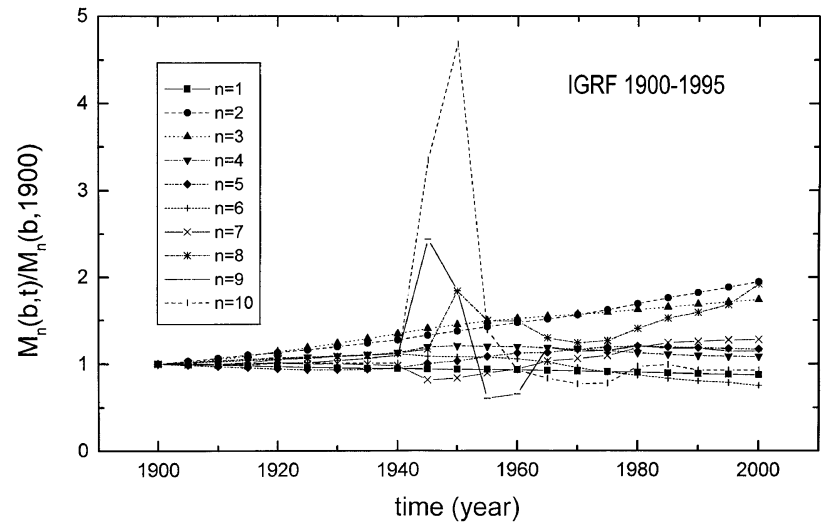

Fig. 3. Time variations of the geomagnetic energy spectra for 1900-2000.

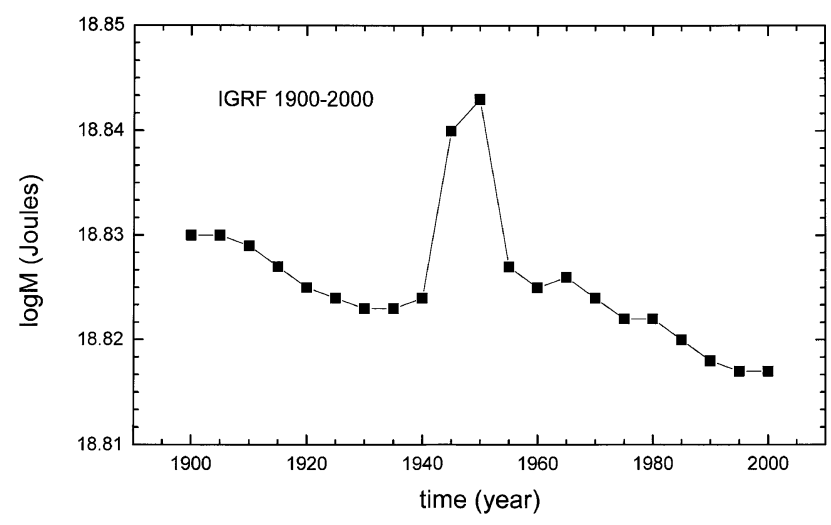

Fig. 4. Total geomagnetic energy vs. time.

in Fig. 3, where $M_{n}(b, 1900)$ is the energy of harmonic of degree $n$ for epoch 1900. In Fig. 3 smooth and consistent secular variations are seen in ratios $M_{n}(b, t) / M_{n}(b, 1900)$ up to $n=5$. A step between 1945 and 1950 is detectable at $n=7$, and becomes more prominent as $n$ increases. The biggest jumps for $n=8,9$, and 10 are respectively 1.837 , 2.439 , and 4.681, compared with approximately 1 before 1940. After 1960 their variations recover smoothly.

In Fig. 4 the total energy $\log M(b, t)$ at the core-mantle boundary is plotted with respect to time. A prominent peak appears at epochs 1945 and 1950 . This spike-like variation is obviously caused by the high-degree $(n=8,9,10)$ Gauss coefficients.

\section{Magnetic Flux at Different Depths}

High-degree Gauss coefficients describe small-scale magnetic configurations. The intensities of these magnetic anomalies on the earth's surface are usually much weaker compared with the low-degree coefficients.

It is noted in the magnetic potential expression Eq. (1) that the importance of a harmonic of degree $n$ is proportional to a ratio $(a / r)^{n+1}$. On the Earth's surface this ratio is 1 for any degree $n$, while on the core-mantle boundary this ratio is about 231, 423, and 774 for $n=8,9$, and 10, respectively. As for energy spectrum, these values are even larger (see Eq. (3)). 


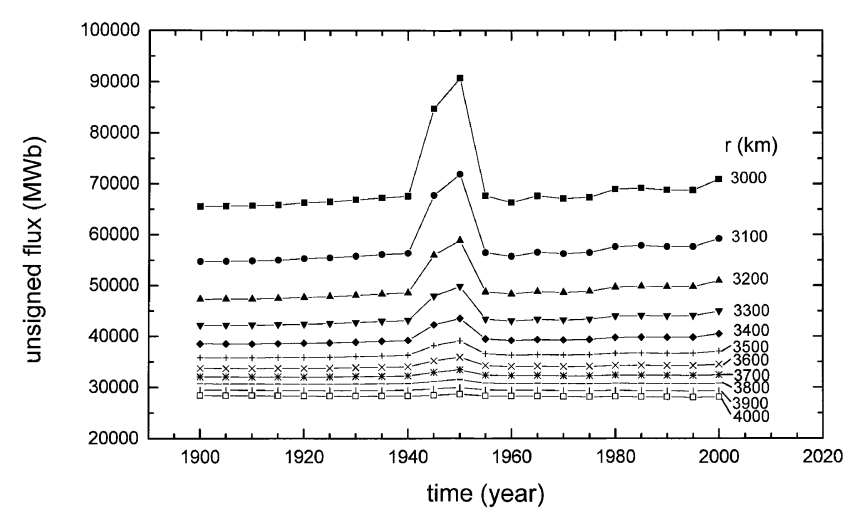

Fig. 5. Variations of magnetic flux at different depths.

Rapid increase of the contribution from high-degree harmonics will greatly affect the magnetic field pattern on the core-mantle boundary and the values of geomagnetic flux in the deep interior.

A useful measure of the strength of the earth's magnetic field is its magnetic pole-strength $P$, the unsigned (or absolute) magnetic flux linking a closed surface $S$ surrounding the earth's core (Voorhies and Benton, 1982). If $S$ is a spherical surface of radius $r$, then

$$
P(r, t)=\int_{0}^{2 \pi} \int_{0}^{\pi}\left|B_{r}(r, \theta, \phi, t)\right| r^{2} \sin \theta d \theta d \phi .
$$

The time variation rate of the flux, $\partial P(r, t) / \partial t$ is commonly used to determine the conducting core radius.

Figure 5 shows the unsigned magnetic flux at different depths (or radius) during 1900-2000. It is noted in the figure that the flux varies very smoothly before 1940 and after 1955 at each of the depths. The variation rates and tendencies during these two periods are consistent. Against these regular variations an unusual jump stands out at epochs 1945 and 1950, and its range enhances as depth increases. It will be interesting to study this kind of unusual short-term variation in detail.

\section{Effect of High-Degree Harmonics on Determi- nation of Core Radius}

The earth's magnetic models are an important data source for studying core dynamics (Bloxham and Gubbins, 1985; Bloxham and Jackson, 1991; Olson and Aurnou, 1999). One of the interesting applications is magnetic determination of the conducting core radius.

If the earth's core is assumed to be perfectly electrically conducting sphere surrounded by the insulating mantle, the magnetic field lines will be frozen in and move together with the core liquid. On the basis of this 'frozen-flux hypothesis', Hide (1978) proposed a method for determining electrically conducting core by extrapolating $\partial P(r, t) / \partial t$ downward through the insulating mantle until the $\mathrm{CMB}$ is reached where $\partial P(r, t) / \partial t=0$. By using this method, Hide and Malin (1981), and Voorhies and Benton (1982) calculated the radius of the conducting core.

Rapid increase of the contribution from high-degree harmonics will greatly affect the values of geomagnetic flux

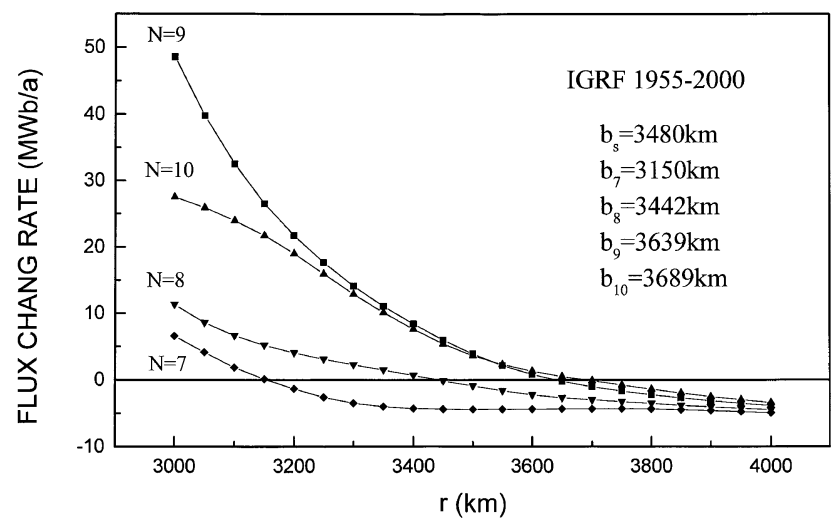

Fig. 6. Earth's core radius determined by geomagnetic field.

$P(r, t)$ and its change rate $\partial P(r, t) / \partial t$ at depth. Consequently, it is expected that the determination of the core radius would be affected by truncation level in the spherical harmonic series of the main geomagnetic field.

As Fig. 5 shows, the unsigned magnetic flux, $P(r, t)$, for each of depths changes at a nearly constant rate during the period of 1900-1940 and 1955-2000. The average change rate for 1955-2000 is used to determine the core radius in this paper. A comparison of the core radii, $b_{N}$, magnetically determined for different truncation level $N$ is shown in Fig. 6. The obtained radii are respectively $3150 \mathrm{~km}, 3442$ $\mathrm{km}, 3639 \mathrm{~km}$, and $3689 \mathrm{~km}$ for $N=7,8,9$, and 10, which are within $9 \%, 1 \%, 4 \%$, and $6 \%$ of the seismic radius $b_{s}$. The truncation of $N=8$ yields the closest value. This result leads to a conclusion that high-degree harmonics in the geomagnetic field expression are important for determining the Earth' core radius. Unfortunately, the accuracy of highdegree coefficients is poor, especially, for 1945 and 1950. It can be seen in Fig. 5 that the change rates for 1945-1950 are positive at all of the depths, while those for 1950-1955 are negative for all of depths. If using the change rate for these two periods, we could not get $\partial P(r, t) / \partial t=0$.

Since the geomagnetic field and its SV models truncated at high degree (for example, $n=13$ ) are usually used to infer the core-mantle boundary flow, the accuracy of these models are essential to geodynamo theory. As Hulot et al. (1992) and Pais and Hulot (2000) showed, the accuracy of the coefficients in the geomagnetic field and its SV models imposes some serious limitation on what can be said about the core flow. A lot of improvement in the higher degree coefficients is still needed.

\section{Discussion and Summary}

The IGRF is compiled on the basis of the candidate models submitted by several agencies or authors (Sabaka et al., 1997). In the IGRF models high-degree Gauss coefficients are usually much smaller than low-degree ones. Their values are comparable to the ranges of the corresponding coefficients in the candidate models. This condition is common at about $n=10$ for the main field and about $n=8$ for the secular variation. As pointed out by Barton (1997), in this case, the coefficients are essentially unresolved. In other words, the high-degree coefficients in the IGRF are not well- 
determined. This is common to every model. However, the above-mentioned irregular behaviour of the high-degree coefficients for 1945-1955 cannot be ascribed to this origin.

The secular variation of the main geomagnetic field is more or less continuous and smooth, although some types of shortterm variations, such as jerks, occur occasionally (Ducruix et al., 1980; Malin et al., 1983). The above-mentioned irregular behaviour of the high-degree coefficients for 1945-1955, however, cannot be explained by the claimed jerks, among which the most credible are those which occurred near 1913, 1970, and 1978.

Between 1900 and 1940, the IGRF varies smoothly, being based on the single time-dependent model GSFC (S95-sc). On the contrary, the models for 1945 onwards were adopted with a view to obtaining the best fit to the available data at discrete 5-year epochs with little or no regard to continuity (Barraclough, 1985, 1987; Langel and Estes, 1987; Langel et al., 1988; Sabaka et al., 1997). This fact leads to a conclusion: the irregular variation of the high degree coefficients for 1945-1955 can be ascribed to the original data which are used to establish the candidate models.

The IGRF models for the 1945-1955 period were adopted in 1985 (Barraclough, 1985, 1987), and their definitive versions in 1987 (Langel et al., 1988). Although the data used to produce the models were collected during and after the war, the modelling work was done in the 1980s for the 4th and 5 th generations of the IGRF. What is mainly tested in this paper is the internal consistency of the IGRF series. I also show the influences of the unusual behaviour of the IGRF during the 1945-1955 period on magnetic flux, spectrum, and the magnetic determination of the core radius. The explanation of the unusual behaviour of the IGRF during the 1945-1955 period requires a re-examination of the candidate models, their original data, and the error analyses. Comparison of the IGRF for the 1945-1955 period with other models (Bloxham and Jackson, 1992) is also necessary. That is our next task and is beyond the scope of this paper.

The results obtained in this paper can be summarised as follows,

(1) The high-degree $(n>7)$ Gauss coefficients of the DGRF models for 1945 and 1950 show some peculiar jumps when compared with models from other epochs, although the low-degree $(n<7)$ Gauss coefficients are consistent.

(2) This unusual behaviour may be ascribed to the data used to create the candidate models, however, a further study is needed.

(3) It seems necessary to re-examine the candidate models of the DGRF 1945 and 1950, check their original data and the error analysis. Comparison of the IGRF for the 1945-1955 period with other models is also needed. Until then a simple approach is linear interpolation between 1940 and 1960 for the $n>7$ Gauss coefficients.

Acknowledgments. The author gratefully acknowledges the contributors to IGRF models. This study is financially supported by the National Natural Science Foundation of China.

\section{References}

Baldwin, R. T. and R. A. Langel, Tables and maps of the DGRF 1985 and IGRF 1990, in IAGA Bulletin, 54, 183 pp., 1993.

Barraclough, D. R., International Geomagnetic Reference Field revision,
Nature, 318, 316, 1985.

Barraclough, D. R., International Geomagnetic Reference Field: the fourth generation, Phys. Earth Planet. Int., 48, 279-292, 1987.

Barton, C. E., International Geomagnetic Reference Field: the seventh generation, J. Geomag. Geoelectr., 49, 123-148, 1997.

Benton, E. R., R. H. Estes, R. A. Langel, and L. A. Muth, Sensitivity of selected geomagnetic properties to truncation level of spherical harmonic expansions, Geophys. Res. Lett., 9, 254-257, 1982.

Bloxham, J. and D. Gubbins, The secular variation of Earth's magnetic field, Nature, 317, 777-781, 1985

Bloxham, J. and A. Jackson, Fluid flow near the surface of Earth's outer core, Rev. Geophys., 29, 97-120, 1991.

Bloxham, J. and A. Jackson, Time-dependent mapping of the magnetic field at the core-mantle boundary, J. Geophys. Res., 97, 19537-19563, 1992.

Cain, J. C., Z. Wang, D. R. Schmitz, and J. Meyer, The geomagnetic spectrum for 1980 and core-crustal separation, Geophys. J., 97, 443-447, 1989.

Chapman, S. and J. Bartels, Geomagnetism, 1049 pp., Oxford University Press, London, 1940.

Ducruix, J., V. Courtillot, and J. L. LeMouel, The late 1960s secular impulse, the eleven year magnetic variation and the electrical conductivity of the deep mantle, Geophys. J. R. Astr. Soc., 61, 73-94, 1980.

Hide, R., How to locate the electrically conducting fluid core of a planet from external magnetic observations, Nature, 271, 640-641, 1978.

Hide, R. and S. R. C. Malin, On the determination of the size of the earth's core from observations of the geomagnetic secular variation, Proc. R. Soc. Lond., A347, 15-33, 1981.

Hulot, G., J. L. Le Mouel, and J. Wahr, Taking into account truncation problems and geomagnetic model accuracy in assessing computed flows at the core-mantle boundary, Geophys. J. Int., 108, 224-246, 1992.

IAGA Commission 2 Working Group 4, International Geomagnetic Reference Field 1965.0, J. Geophys. Res., 74, 4407-4408, 1969.

IAGA Division 1 Study Group on Geomagnetic Reference Field, International Magnetic Reference Field 1975, J. Geomag. Geoelectr., 27, $437-$ 439, 1975.

IAGA Division 1, Working Group 1, International geomagnetic reference field: DGRF 1965, DGRF 1970, DGRF 1975 and IGRF 1980, EOS Trans. Am. Geophys. Union, 62, 1169-1170, 1981.

IAGA Division 1, Working Group 1, International geomagnetic reference field revision 1985, J. Geomag. Geoelectr., 37, 1157-1163, 1985.

IAGA Division 1, Working Group 1, International geomagnetic reference field revision 1987, J. Geomag. Geoelectr., 39, 773-779, 1987.

IAGA Division 5, Working Group 8, International Geomagnetic Reference Field, 1991 revision, J. Geomag. Geoelectr., 43, 1007-1012, 1991.

IAGA Division 5, Working Group 8, International Geomagnetic Reference Field, 1995 revision, Geophys. J. Int., 125, 318-321, 1996.

IAGA Division 5, Working Group 8, International Geomagnetic Reference Field 2000, Geophys. J. Int., 141, 259-262, 2000.

Langel, R. A., The main field, in Geomagnetism, edited by J. A. Jacobs, Vol. 1, pp. 249-512, Academic Press, London, 1987.

Langel, R. A., International geomagnetic reference field: the sixth generation, J. Geomag. Geoelectr., 44, 679-707, 1992.

Langel, R. A. and R. H. Estes, A geomagnetic field spectrum, Geophys. Res. Lett., 9, 250-253, 1982.

Langel, R. A. and R. H. Estes, Derivation of proposed International Geomagnetic Reference Field models for 1945, 1950, 1955, and 1960, Phys Earth Planet. Int., 48, 293-305, 1987.

Langel, R. A., D. R. Barraclough, D. J. Kerridge, V. P. Golovkov, T. J. Sabaka, and R. H. Estes, Definitive IGRF models for 1945, 1950, 1955, and 1960, J. Geomag. Geoelectr., 40, 645-702, 1988

Lowes, F. J., Mean square values on sphere of spherical harmonic vector field, J. Geophys. Res., 71, 2179, 1966.

Lowes, F. J., Spatial power spectrum of the main geomagnetic field and extrapolation to the core, Geophys. J. R. Astr. Soc., 36, 717-730, 1974.

Macmillan, S. and C. E. Barton, Magnetic field maps for IGRF 1995, J. Geomag. Geoelectr., 49, 149-156, 1997.

Malin, S. R. C., B. M. Hodder, and D. R. Barraclough, Geomagnetic secular variation: A jerk in 1970. Publicado en volumen commemorativo 75 aniversario del observatorio del Ebro, (75th anniversary volume of Ebro observatory), edited by J. O. Cardus, pp. 239-256, Tarragona, 1983.

Olson, P. and J. Aurnou, A polar vortex in the Earth's core, Nature, 402, 170-173, 1999.

Pais, A. and G. Hulot, Length of day decade variations, torsional oscillations and inner core superrotation: evidence from recovered core surface zonal flows, Phys. Earth Planet. Int., 118, 291-316, 2000. 
Sabaka, T. J., R. A. Langel, R. T. Baldwin, and J. A. Conrad, The geomagnetic field 1900-1995, including the large-scale field from magnetospheric sources, and the NASA candidate models for the 1995 revision, J. Geomag. Geoelectr., 49, 157-206, 1997.

Verosub, K. L. and A. Cox, Changes in the total magnetic energy external to the earth's core, J. Geomag. Geoelectr., 23, 235-242, 1971.

Voorhies, C. V. and E. R. Benton, Pole-strength of the Earth from MAGSAT and magnetic determination of the core radius, Geophys. Res. Lett., 9 , 258-261, 1982.

Zmuda, A. J., The International Geomagnetic Reference Field, 1965.0, in IAGA Bulletin, 28, World Magnetic Survey, IUGG, Paris, 1971.

W.-Y. Xu (e-mail: wyxu@mail.c-geos.ac.cn) 